\title{
Developing a Mining Robot for Mars Exploitation: NASA Robotics Mining Competition (RMC)
}

\author{
Tariq Tashtoush*1, Agustin Velazquez ${ }^{2}$, Andres Aranguren ${ }^{3}$, Cristian Cavazos ${ }^{4}$, David Reyes ${ }^{5}$, Edgar Hernandez ${ }^{6}$, \\ Emily Bueno $^{7}$, Esteban Otero ${ }^{8}$, Gerardo Zamudio ${ }^{9}$, Hector Casarez ${ }^{10}$, Jorge Rullan ${ }^{11}$, Jose Rodriguez ${ }^{12}$, \\ Juan Carlos Villarreal ${ }^{13}$, Michael Gutierrez ${ }^{14}$, Patricio Rodriguez ${ }^{15}$, Roberto Torres ${ }^{16}$, \\ Rosaura Martinez ${ }^{17}$, and Sanjuana Partida ${ }^{18}$ \\ School of Engineering \\ Texas A\&M International University \\ Laredo, TX, 78041 USA
}

\begin{abstract}
This paper focuses on demonstrating the design and build stages, and effort done by Systems Engineering students team (DustyTRON NASA Robotics) to develop a mining robot that was used in the 2016 National Aeronautics \& Space Administration (NASA) Robotics Mining Competition (RMC). The objective of the NASA RMC challenge is to encourage engineering students to design and build a robot that will excavate, collect, and deposit a simulated Martian regolith. Mining water/ice, and regolith is very essential task for space missions and resource utilization, they contain many elements such as metals, minerals, and other compounds. The Mining will allow extracting propellants from the regolith such as Oxygen and Hydrogen that can be used as an energy source for in-space transportation. In addition, the space mining system can be used in tasks that are important for human and robotics scientific investigations. The DustyTRON team consists of Systems Engineering students, who are divided into 1) hardware design, 2) electrical circuitry and 3) software development sub-teams. Each team works in harmony to overcome the challenges had previously experienced, such as heavy weight, circuitry layout design, autonomous and user control modes, and better software interface. They designed and built a remote controlled excavator robot, that can collect and deposit a minimum of ten (10) kilograms of regolith simulant within 15 minutes. The developed robot with its innovative mining mechanisms and control system and software will assist NASA in enhancing the current methodologies used for space/planet exploration and resources' mining especially the Moon and Mars. NASA's going-on project aims to send exploration robots that collect resources for analysis before sending astronauts. In 2016, only 56 United State (US) teams were invited to participate, and DustyTRON was one of three university teams from the state of Texas, the team placed the 16th in overall performance. This paper will address the full engineering life-cycle process including research, concept design and development, constructing the robot and system closeout by delivering the team's robot for the competition in Kennedy Space Center in Florida.
\end{abstract}

Keywords-NASA Robotics Mining competition; mining robot; ice regolith; autonomous; NASA space exploration; systems lifecycle; mechanical structure design; control system; systems engineering

\section{INTRODUCTION}

National Aeronautics and Space Administration (NASA) in the leader in space exploration the first robot landed on the moon. These robots were developed to be unmanned and will be the first to explore and mine resources from habitats that humans can not explore either due to high associated cost or highly hazardous planets ecosystem [1-11]. Robotics will allow humans to explore planets surfaces and resources while keeping high level of astronauts safety and lower costs to transport human to space. Additionally, robots will be capable of mining all the minerals and underground resources that will provide the needed energy (Oxygen and Hydrogen).

The NASA Robotic Mining Competition (RMC) encourages university students in the United States to be innovators and creative thinkers to design, build, and compete with robots that can traverse the simulated Martian chaotic terrain; then excavate the basaltic regolith simulant (called Black Point-1 or BP-1) and the ice simulant (gravel), which are a representation of the necessary resource on Mars and return the excavated mass for a deposit into the collector bin to simulate an offworld mining mission.

On May 16-20, 2016, the seventh annual NASA Robotic Mining Competition was held at the Kennedy Space Center in Florida. This event brings together student teams from universities across the US to compete in a real of robotics, remote operation, and automation challenge related to NASA missions. Texas A\&M International University (TAMIU) DustyTRON Robotic team, known as DustyTRON 2.0, worked to fulfill the competition goals, according to NASA's systems engineering guidelines and NASA RMC requirements [12-14]. This will mark the second entry into such competition and the team decided to build a new robot design from the ground up.

This paper is a detailed systematic engineering analysis and design process of DustyTRON 2.0 robot, where a fully functional mining robot will be constructed to fit certain specification including size dimension $(1.5 \mathrm{~m} * 0.75 \mathrm{~m} * 0.75 \mathrm{~m})$, maximum weight $(80 \mathrm{Kg})$, and mechanism (traverse, excavate and deposit). The paper will be centered on the team's design theory and Quality Function Deployment (QFD) analysis. Multiple designs are being evaluated according to preset criteria such as design to build, mobility, weight and budget. Then, one design will be chosen and analyzed in depth.

DustyTRON 2.0 had been divided into three sub-teams: 1) hardware design and construction, 2) electrical circuitry design, and 3) software development, in which the seventeen team members and there assignments is distributed as shown in Fig. 1. 


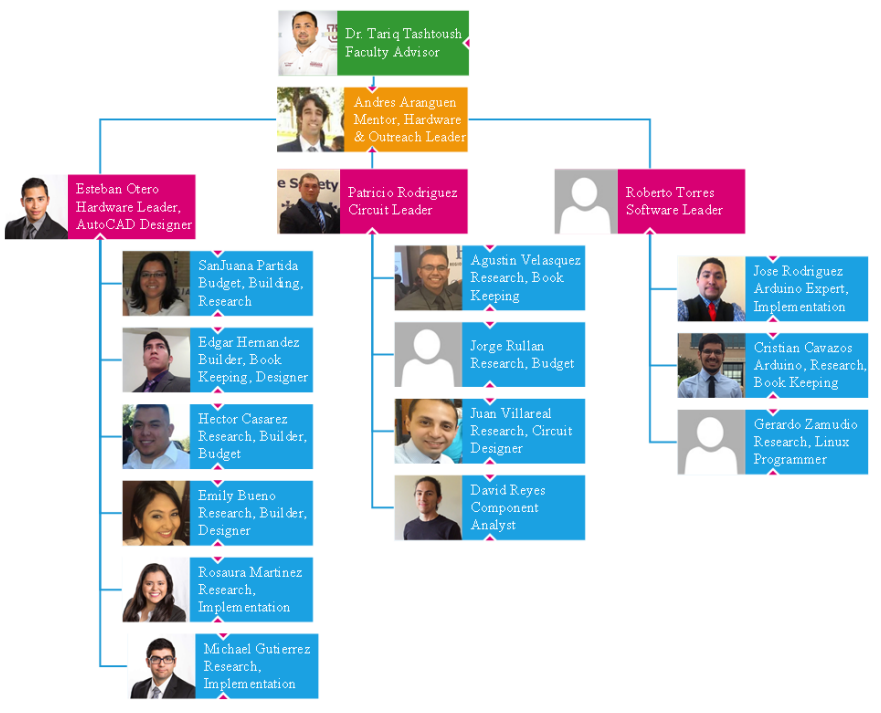

Fig. 1. DustyTRON 2.0 Team Management

The hardware design and construction team will be focusing on building a strong robot structure that can be moved easily keeping light weight while having an excavation, a regolith deposit and the moving mechanisms. The electrical Circuitry team is the bridge that links hardware and software together to make the robot functional. They will improve DustyTRON circuitry design to make sure that the components are easier to access. Cables will also be easily traceable in case of troubleshooting, thus the number of components required. While, the software development team intend on achieving autonomous functionality on DustyTRON 2.0 by utilizing the capability of a System-on-Chip (SoC) and microprocessor systems, where both systems will be communicating through serial interface. They will be using secure connection between the robot and the control station, while using Open-CV (Computer Vision) library for image and object detection to help achieving the autonomous mode. In addition, software development team will have to create an easy access and monitoring connection to the SoC system for rapid maintenance and troubleshooting immediately if errors occur during autonomous mode.

DustyTRON NASA robotics team participated in the NASA RMC 2015, with their mining robot DustyTRON 1.0, as shown in Fig. (2 and 3). There were several major issues with "DustyTRON 1.0" robot design that needed to be addressed and improved upon for the new design.

The first issue was the weight of the robot, DustyTRON 1.0 was borderline on the $80 \mathrm{~kg}$, the weight limit requirement for the NASA RMC competition. That heavy weight altered the robot's ability to move efficiently around the simulated terrain. For this reason, this year we only considered using only light materials when constructing the frame, the excavation mechanism, and the wheel system. DustyTRON 2.0 will be having lighter materials, a simpler frame, and lighter motors.

Second issue was DustyTRON 1.0's steering system, it was not very efficient and resulted in limited maneuverability and agility which diminished the amount of runs the team was able to make during the allowed ten (10) minutes. The steering and

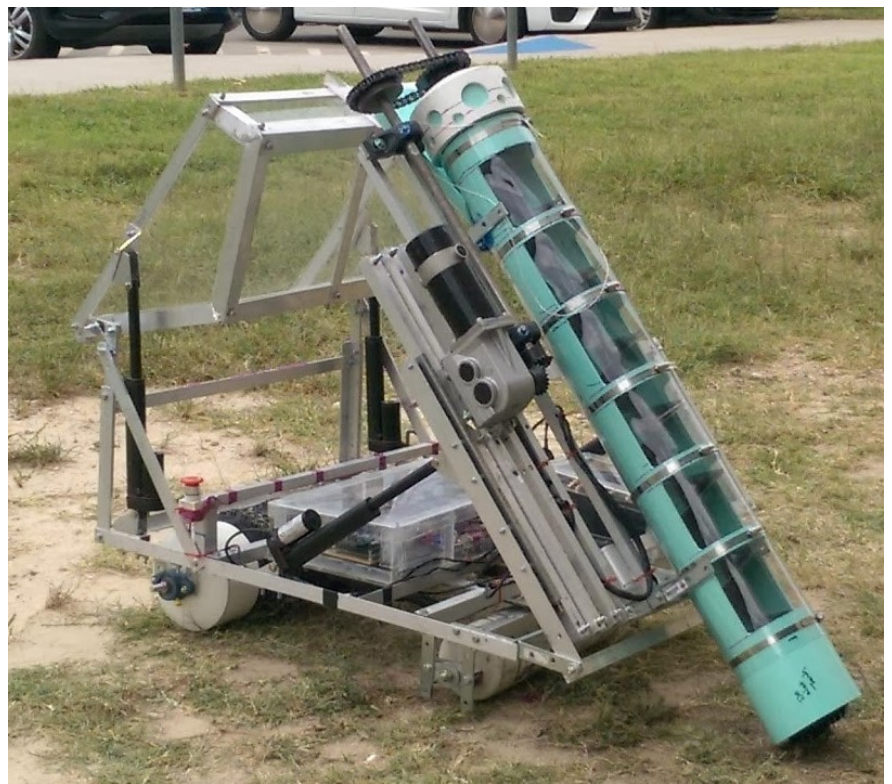

Fig. 2. DustyTRON 1.0 Robot

wheel system included a tube shaped wheel that had a limited turning angle and because of the tube's large surface area, and it had high friction due to large ground contact area. In addition, it had limited ability to drive over rocks and get out of any potential ditches.

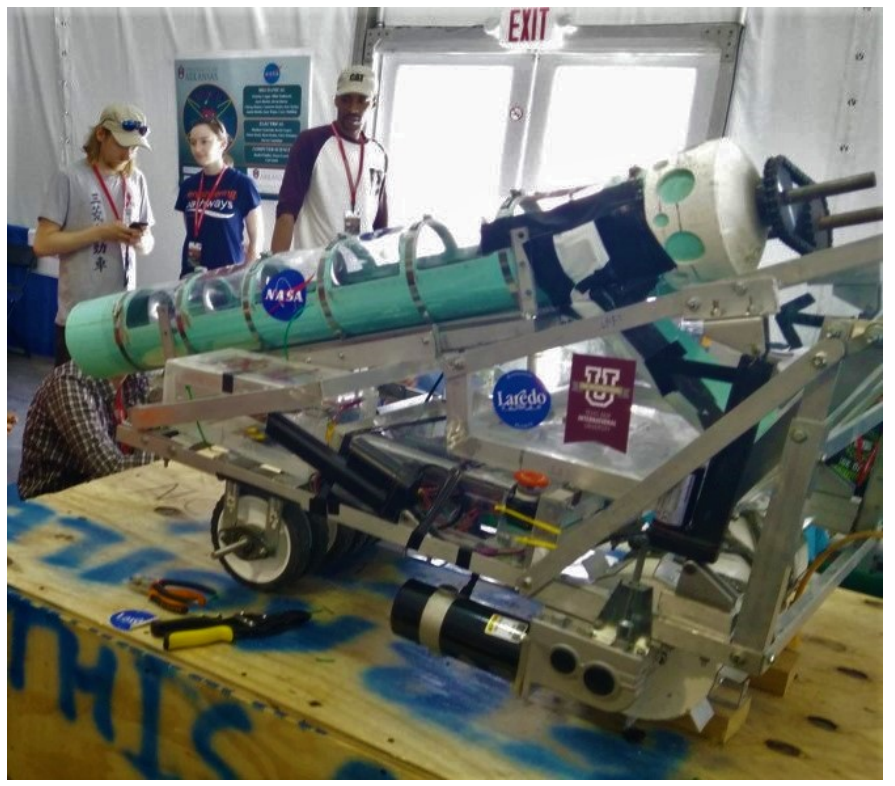

Fig. 3. DustyTRON 1.0 Robot Side-View

DustyTRON 2.0 will have an improved steering and wheels system by using four individual wheels were chosen as the new driving system with bigger tires that will be easier to manipulate and will traversed more efficiently through the simulated Martian terrain. These wheels would give DustyTRON 2.0 a clearance of six (6) inches from the ground to the lower digging mechanism (auger) tip and eight (8) inches for the frame while having the digging capability to adjust as desired 
using the adaptive suspension system. This space would be key in allowing the robot to drive over any potential small to medium sized rocks. Secondly, in conjunction with the software team, the circuitry team will be able to power and control each wheel-motor individually and independently, and adding better overall performance.

DustyTRON 1.0's upper frame was not secured enough and it would tremble excessively when the auger would excavate due to the fact of being top heavy, as shown in Fig. (4). This compromised the structural integrity of the whole robot. The DustyTRON 2.0 design will have a more stable frame that will allow it to remain stationary as the auger excavates and collects the regolith. Another improvement made to the newer design was to add a conveyor belt with scoops that would serve as both the collecting and dumping mechanism of the robot. This will reduce the weight of the robot and will simplify the control system.

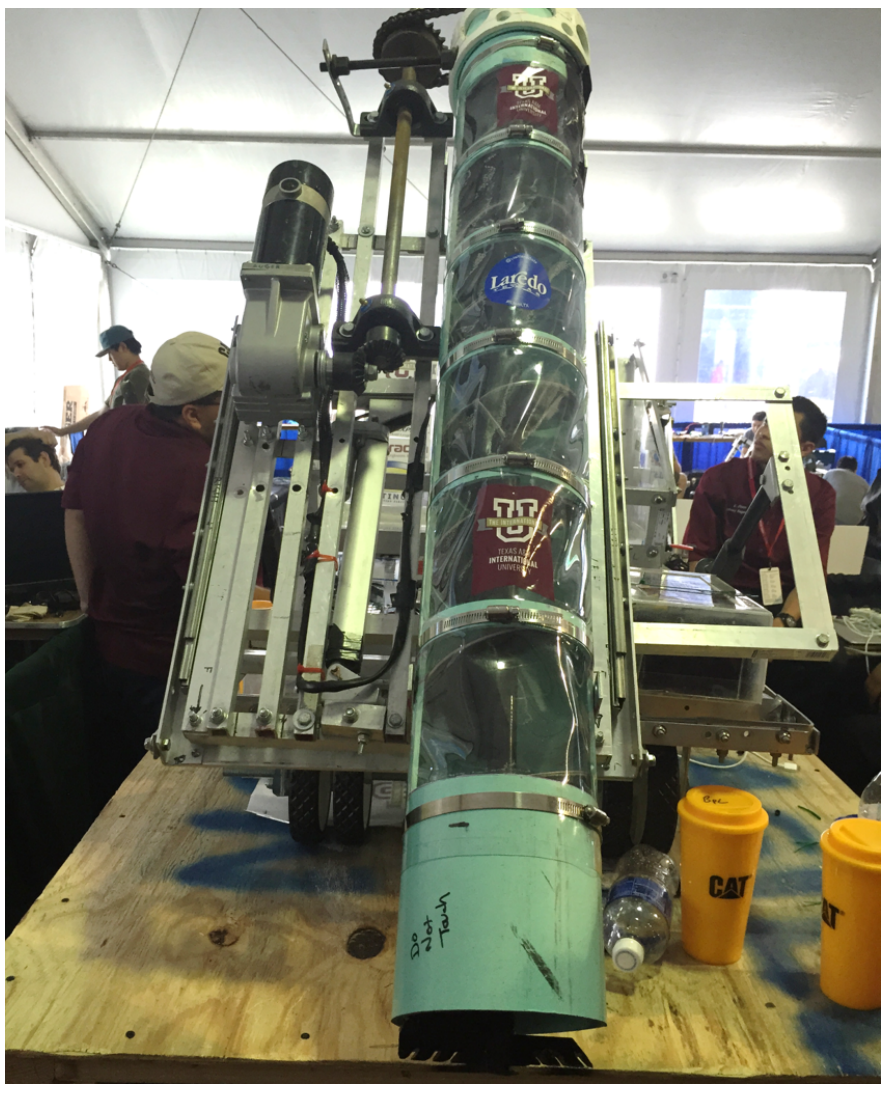

Fig. 4. DustyTRON 1.0 Robot Front-View

The electrical components in DustyTRON 1.0 robot were protected by a box made of Plexiglas, which was located under the excavation mechanism. This made it difficult to access, modify, or replace any of the components without repositioning the robot. Another drawback of the design was the way in which components were placed near areas of robot high activity, where many parts move directly over and regolith passing above it. DustyTRON 2.0 design of the box will be made more ergonomic. The electrical layout and connections are being placed in a more organized manner. The overall circuit design had been improved to include electrical box located at an elevated position and away from moving components, such as the auger and dumping mechanism. The placement at a higher point gives the DustyTRON 2.0 team easier access for maintenance, troubleshooting, or parts replacement.

DustyTRON 1.0 robot was controlled remotely using two Xbox 360 controllers that were used to control directional movement and the excavation mechanism separately. DustyTRON 2.0 will attempt the autonomous control with the option of manual control with two controllers. Another addition to DustyTRON 2.0 are optical sensors such as a Microsoft Xbox One Kinect and a rear local network (IP) servo-controlled camera. These will allow the robot to work autonomously which will require less human intervention and less bandwidth usage. The Kinect camera is installed in the front part of the robot to allow it to scan the environment. The rear servo-camera is installed to allow the monitoring and regulation of both collection and deposit mechanism. These cameras are new components that DustyTRON 1.0 did not possess, it only relied on manual control and simulated terrain cameras. In summary, these components will allow DustyTRON 2.0 to be independent, achieve autonomous, manual control and use less bandwidth and power.

The team felt there were opportunities to improve our robot's functionality and coding by cleaning up the Arduino code. This simplified the code and facilitate the command and communication processes. Another way that DustyTRON 2.0 is trying to fulfill the requirements of being autonomous mode is by using the Jetson TK1. This will aid in wireless communication and computer vision processing capability via the Graphics Processing Unit (GPU). Software Team worked together when coding the Arduino in order to ensure everyone would have their input in the coding and understand when shifting the workload between the Arduino Mega and the Jetson TK1.

DustyTRON 2.0 team cleaned up the coding of the manual controlling Visual-Basic code. The manual mode will be utilized in the event that the Xbox camera would not function properly for the image detection, object avoidance or in the event of any other connectivity issues. The software team is planning to establish an algorithm for the usage of the Xbox 360 wired controllers. The establishment and application of these algorithms will allow for full control of the robot's wheel motors with the controller. It will also provide direct commands to the robot's functions.

The paper is organized as follows: Section 2 is short history of NASA mission to explore the Moon and Mars and how it was done, Section 3 a summary of system requirements and the team's preliminary Designs, Section 4 describes concept operation, Sections 5 illustrates the system hierarchy, while Section 6 Risk management, trade-off analysis, verification of System Meeting Requirements and reliability, Section 7 summarises the results of the competition, and Section 8 concludes the paper and describe the team future plan.

\section{LiterATURE REVIEW AND NASA RMC History}

This section aims to introduce and explain how the NASA Robotic Mining Competition (RMC) works. Recent discoveries by NASA missions to Mars have found large amounts of water in the form of water ice and hydrated minerals on Mars utilizing the space rovers such as "Curiosity" and orbiting 
satellites [12-14]. These sources of water resulted from clay and clay-like minerals that formed millions years ago on the surface or underground of Mars. Capturing this water is key to allow humans to "live off the land" by utilizing the available resources. The water can be used for human use, hygiene, rocket propellant, growing plants, radiation shielding, and can be used in various other processes. in order to gain access to that water, minerals that contain the water must be mined out and the surface soil "regolith" be removed to expose the water resources.

The NASA Robotic Mining Competition is for universitylevel undergraduate students to challenge them to design and build a mining robot that can traverse simulated Martian terrain. The mining robot must excavate the regolith simulant and/or the ice simulant and return and deposit the excavated mass to a collection bin from a space station. The challenge contains few complexities such as the complexities the abrasive characteristics of the regolith, the limited robot weight/size and the required tele or autonomous operation of the the robot from a remote Mission Control Center. Additionally, participating teams must consider a number of design and operation factors such as dust tolerance and dust projection, mass, communications/energy/power consummation and autonomy.

NASA benefits from the competition by encouraging student teams to develop innovative robotic excavation concepts, which may lead to a creative ideas that can be used in an actual excavation device for NASA future missions. Advances in Martian mining have the potential to significantly contribute to human spaceflight and NASA space exploration operations. Details of this competition can be found online at https://www.nasa.gov/nasarmc.

The NASA RMC started in its original format in 2010 as NASA Lunabotics Competition [13]. In 2011, it was open to undergraduate and graduate student teams enrolled in colleges or universities worldwide. But in 2014, due to NASA budgetary constraints, the competition was limited to teams from United States colleges or universities. In 2020, NASA transited to a Lunar focused competition, and Table I represent the competition year, name and the allowed countries to participate.

TABLE I. NASA RoBotics Mining COMPETITION HistoRY

\begin{tabular}{|l|l|}
\hline Competition Year and Name & Competition Participants \\
\hline (2010) Lunabotics & USA \\
\hline (2011) Lunabotics & $\begin{array}{l}\text { USA, Bangladesh, Canada, Colombia, } \\
\text { India, Spain }\end{array}$ \\
\hline (2012) Lunabotics & $\begin{array}{l}\text { USA, Bangladesh, Canada, Colombia, } \\
\text { India, Mexico, Romania, South Korea }\end{array}$ \\
\hline (2013) Lunabotics & $\begin{array}{l}\text { USA, Australia, Bangladesh, Canada, } \\
\text { Colombia, India, Mexico, Poland }\end{array}$ \\
\hline (2014-2019) RMC & USA \\
\hline (2020-present) RMC: Lunabotics & USA \\
\hline
\end{tabular}

Many teams allover the United state presented in the robotics design they came up with and met the NASA RMC requirements [15-22]. In this paper DustyTRON team is presenting their robot, the design process and their achievements in the 2016 NASA RMC. This paper will provide an insight for other researchers and teams to follow the system engineering concepts and process to develop new technologies and designs that can improve the human race to explore and populate space.
This paper is a successful example of utilizing and implementing systems engineering concepts to real-life problems and industries not traditionally known for the use of systems engineering.

\section{SyStEM REQUIREMENTS AND PRELIMINARY DESIGNS}

The purpose of the project is to create an inexpensive improved rover system that can perform multiple functions, such as image capturing, rock mining, and data collection while exploring the space. Many researchers and engineering teams [23-34] worked on developing new technologies that can help to explore beyond our earth mainly Mars and the moon.

\section{A. System Requirements}

To begin the design process, the team gathered the requirements that will benchmark their design. These requirements were derived from the NASA RMC competition rules and regulations, and it meant to ensure meeting the competition needs and goals. The team broke the system down into functional subsystems and identified how they would interact. Then, the team generated concepts for each subsystem, scoring them against the requirements to determine the final design. The key requirements are listed in Table II.

TABLE II. SYSTEM REQUIREMENTS EXTRACTED FROM [23-25]

\begin{tabular}{|c|c|c|}
\hline $\begin{array}{l}\text { Requirement } \\
\text { Type }\end{array}$ & Action & Specifications \\
\hline \multirow[t]{3}{*}{$\begin{array}{l}\text { Performance } \\
\text { Requirements }\end{array}$} & $\begin{array}{l}\text { Regolith: } \\
\text { Excavate }\end{array}$ & $\begin{array}{l}\text { Have an excavation mechanism that will be able } \\
\text { to excavate an adequate depth that will reach the } \\
\text { ice simulant }\end{array}$ \\
\hline & $\begin{array}{l}\text { Regolith: } \\
\text { Collect }\end{array}$ & $\begin{array}{l}\text { The robot must be equipped with a form of } \\
\text { storage that it will use to collect the regolith } \\
\text { excavated }\end{array}$ \\
\hline & $\begin{array}{l}\text { Regolith: } \\
\text { Deposit }\end{array}$ & $\begin{array}{l}\text { The robot must be capable of depositing the } \\
\text { regolith collected onto a bin located at the back } \\
\text { of the simulated terrain }\end{array}$ \\
\hline \multirow[t]{2}{*}{$\begin{array}{l}\text { Design } \\
\text { Requirements }\end{array}$} & Dimensions & $\begin{array}{l}\text { The robot must not exceed the measurements of } \\
1.5 \mathrm{~m} \text { in length, and } 0.75 \mathrm{~m} \text { in both height and } \\
\text { width }\end{array}$ \\
\hline & Weight & $\begin{array}{l}\text { The robot must weigh less than } 80 \mathrm{Kg} \text { in order } \\
\text { to compete }\end{array}$ \\
\hline
\end{tabular}

\section{B. DustyTRON 2.0 Preliminary Designs}

1) Design \#1: The first robot design shown in Fig. 5, illustrate a robot that will excavate the simulated Martian regolith using two augers and store the regolith into a Plexiglas box, as shown in the AutoCAD design. This design will feature a frame that will be built using aluminum flat bars and angles and with PVC pipes to cover the augers. The chassis design is $1 \mathrm{~m}$ in length and $0.5 \mathrm{~m}$ in width.

The chassis will contain two Plexiglas boxes, one for the micro-controller, batteries, motors drivers and other electronics compartment, while the second box is for regolith collection behind the auger system. The four actuators, suspension, and wheels will be mounted to the chassis. Front wheels will be four inches in diameter and an inch wide, while rear wheels will be ten inches in diameter and four inches wide.

The auger system consist of two augers that will have a length of $0.513 \mathrm{~m}$, and each auger will be housed in a $0.152 \mathrm{~m}$ inner diameter PVC pipe. The augers will be 
attached to the chassis, which acts as the base of the robot, and will be manipulated by the moving of the four cornered actuators/suspensions. The system will be powered with one 24V 280W motor.

The team decided to make the rear wheels larger and wider to help the robot maneuver more efficiently on the simulated Martian terrain. Two augers were added in order to increase the amount of regolith that could be excavated during the allowed ten minutes. The team decided to utilize Plexiglas again for the electrical box since it is durable and lightweight material, and has been proven to be able to protect the electrical components quite efficiently. The location of the box was also a major concern, as it needed to be easily accessible.

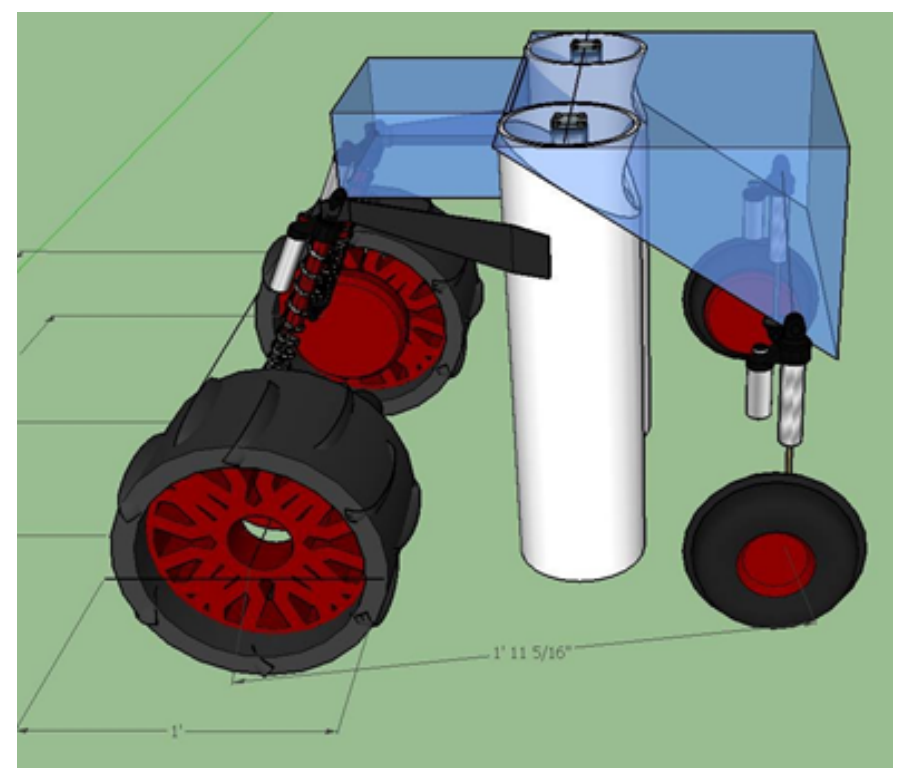

Fig. 5. DustyTRON 2 Mechanical Structure AutoCAD Design 1

2) Design \#2: The structure for design 2 is very similar to that of Design 1 and shown in Fig. 6, except for a few changes that team members felt that they were necessary. Firstly, the team decided to only use one auger. This was in part due to the fact that one auger would still excavate an adequate amount of regolith, and the addition of another auger would only increase the power consumption. Another component that differs from Design 1 is the wheel system, which consists of six wheels that will be eight inches in diameter and six inches wide. The team also decided that the center wheels will be the only ones with a motor, this wheel setup requires two $12 \mathrm{~V}$ motors.

3) Design \#3: The third design structure is similar to the prior designs, except for a few differences. The number of wheels was decreased to four, and the number of actuators was decreased to only two, unlike the last two designs which included four actuators. The size of the wheels will be larger in order to provide better traction and mobility to the robot. Also, the collection bin will be reinforced with a metal frame in order to attach both a camera and emergency stop buttons. The illustration for this design is show in Fig. 7.

4) Design \#4: For design 4 illustrated in Fig. 8, the upper structure underwent significant modifications, mainly the use of a new lifting mechanism. The team decided to implement

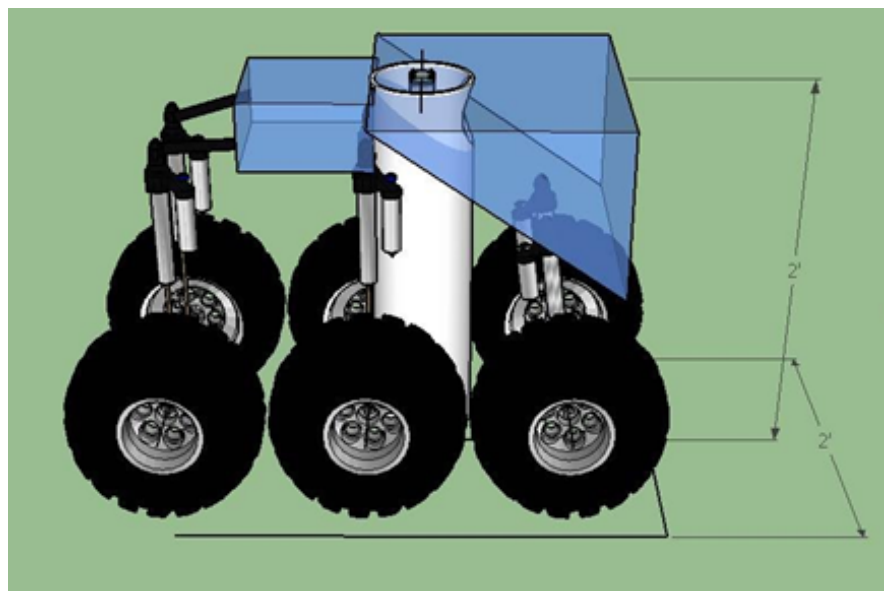

Fig. 6. DustyTRON 2 Mechanical Structure AutoCAD Design 2

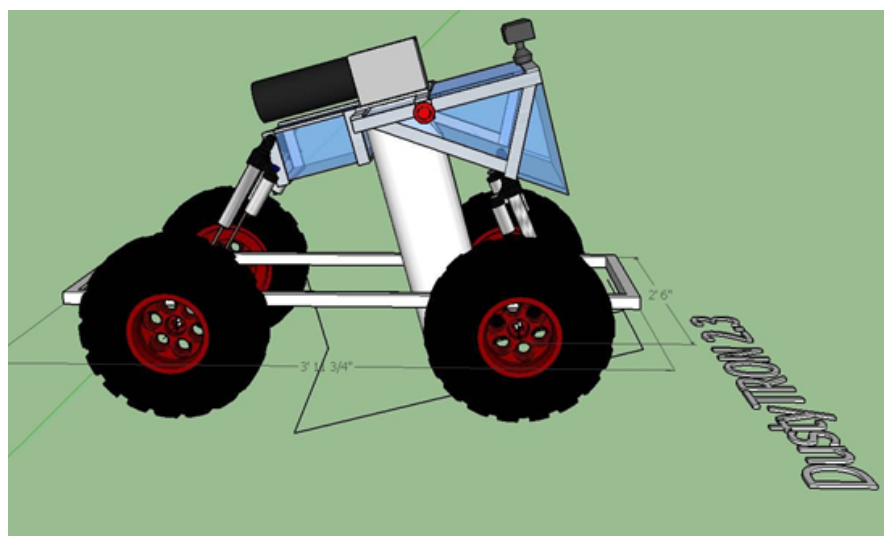

Fig. 7. DustyTRON 2 Mechanical Structure AutoCAD Design 3

a scissors-lift mechanism to the robot, instead of actuators as a mean to lift the upper structure. This design will allow the robot to be able to excavate deeper, but it will also have to support all of the robot components excluding the wheels. The number of actuators for this design remained as two. All other aspects of the design were the same as previous designs including the wheels, collection bin, camera, and the electrical components box.

5) Design \#5: Final Design: This design underwent various major changes, the first and most important one is the addition of a slider to the excavation mechanism. This means the auger, and the powering motor will be mounted on a sliding mechanism that will allow for deeper reach. Another major change was the to change the frame materials from aluminum bars to 80/20 T-slotted extrusion bars. This material was the only material used to build both the frame and the sliding mechanism that would hold the auger and its motor. Another major addition was a conveyor belt equipped with scoops that will act as both a collecting box and dumping mechanism. The belt will collect the dirt and hold it until an adequate amount is collected, then it will deliver the regolith to the collecting bin. This ultimately became the final design that was built because of many reasons, the most important one being the weight. The t-slotted bars are both strong and extremely lightweight, making the frame both stable and light. Also, the 


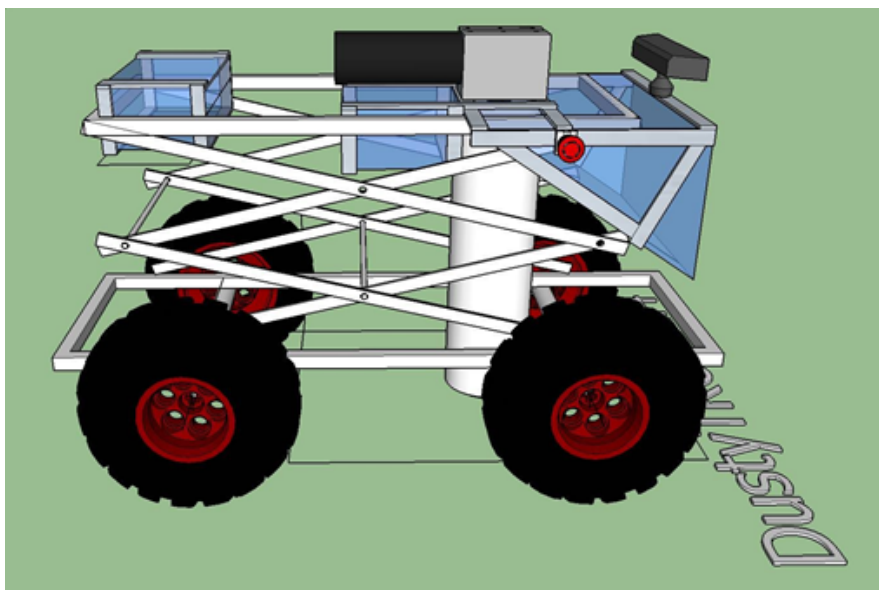

Fig. 8. DustyTRON 2 Mechanical Structure AutoCAD Design 4

sliding mechanism proved to be a better option to increase the depth of the excavation without compromising the integrity of the frame. This design is shown in Fig. 9.

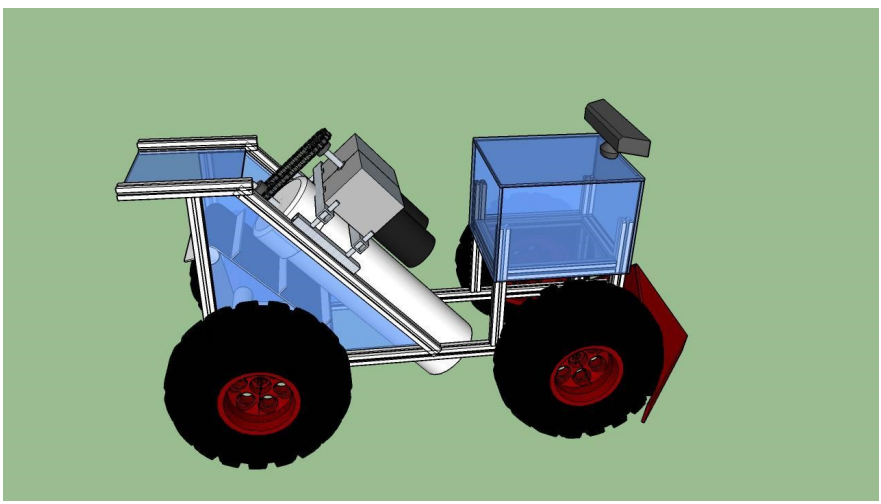

Fig. 9. DustyTRON 2 Mechanical Structure AutoCAD Design 5

Per DustyTRON 2.0 design, the system required four independently moving wheels and one 6 inches auger to excavate. These requirements laid out the fundamental ideas of circuit design. It was immediately recognized that four $12 \mathrm{~V}$ motors were going to be required for the wheels as well as one $24 \mathrm{~V}$ motor for the auger, these requirements helped in defining the power distribution setup. Using $12 \mathrm{~V}$ motor and motor drivers in the testing stage, showed the power was not enough to rotate the auger at the desired speeds. Therefore, circuit team decided to use the same batteries connection as DustyTRON 1.0 but with the option of having both $12 \mathrm{~V}$ and $24 \mathrm{~V}$ outputs. Working with four batteries, two pairs would be connected in series and the pairs would then be connected in a parallel configuration, which will provide each component with the appropriate voltage, either $24 \mathrm{~V} / 14 \mathrm{~A}$ or $12 \mathrm{~V} / 7 \mathrm{~A}$.

DustyTRON 2.0 design required different software configuration and code that will make a simpler operation. To achieve that, Arduino codes have been reformed (easier to read and user-friendly) to make executable commands easier and more precise. This is a significant improvement over the previous DustyTRON Arduino code, which was too complex and only readable to DustyTRON 1.0's programmers. Software team worked collectively to code Arduino and each of its individual parts to be readable to every team member. In addition, Arduino open source libraries will be used for each of the individual components of the robot, such as Victor motor drivers, Jaguar motor drivers, and Axis 206 local network (IP) Camera are all using the Servo library from Arduino. While the Pololu motor driver will be using last year's library to operate the auger motor and the linear actuators.

\section{CONCEPT OPERATIONS}

DustyTRON 2.0 must meet the required performance of the RMC and outperform last year's robot. Its frame will be substantially lighter than the allotted $80 \mathrm{Kg}$. The frame is constructed using 80/20 T-slotted extrusion bars, which is lightweight and will be used for different aspects of the robot. To excavate the required BP-1 plus the gravel (icy regolith simulant); DustyTRON 2.0 is using a shorter auger, which is powered with two 24V CIM motors with gear box of $47: 1$ ration.

DustyTRON 2.0 will feature a double spiral auger to increase productivity in the same amount of time. It will also be operated autonomously through multiple cameras, microprocessor and graphical processing unit, and if that fails, it will be manipulated over WiFi by two controllers. The robot will also incorporate a conveyor belt that will store then transport the collected BP-1 into the deposit bin. This will help in simplifying the collecting process by reducing the auger movement, and eliminate the storage box. Also, four $30 \mathrm{~cm}$ wheels will help to overcome any obstacle in the simulated Martian terrain while a front guard will also protect all the component under the robot.

\section{System Hierarchy}

To effectively visualize the main components of DustyTRON 2.0 robotics and their tasks and relation to each other, a system hierarchy was mapped out for each sub-team. The hardware components' hierarchy is showing in Fig. 10, it represent the mechanical components that dictate the robot structure integrity and performance. Robot structure consist of the wheels (for linear motion and steering), linear actuators (for individual excavation, collection and depositing components movements and suspension/steering system) and Motors.

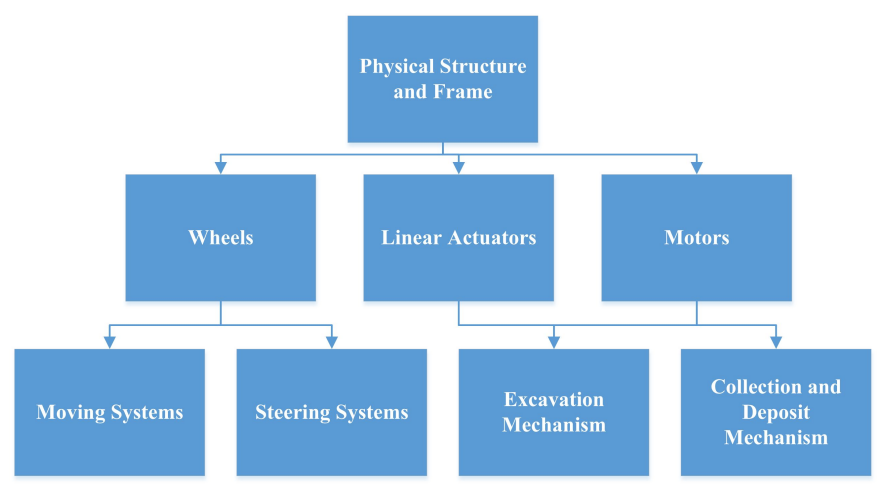

Fig. 10. Hardware System Hierarchy for DustyTRON 2.0 
For the electrical circuit and software sub-teams, many parts are common between them. For manual control, a Microsoft Xbox 360 controller sends command inputs through the controller computer, which is wireless connected to the NVIDIA Jetson TK1 that's embedded in DustyTRON 2.0. For autonomous mode, a Microsoft Xbox One Kinect feeds image and video data to the TK1 for objects detection purposes, while a rear servo camera feeds image and video data to the TK1 in regards to regolith deposit. Whether it be manual or autonomous mode, TK1 will use the provided input to command to the Arduino Mega, which will be directly controlling the motor drivers. These motor drivers will power all motors to achieve the desired movements as shown in Fig. 11.

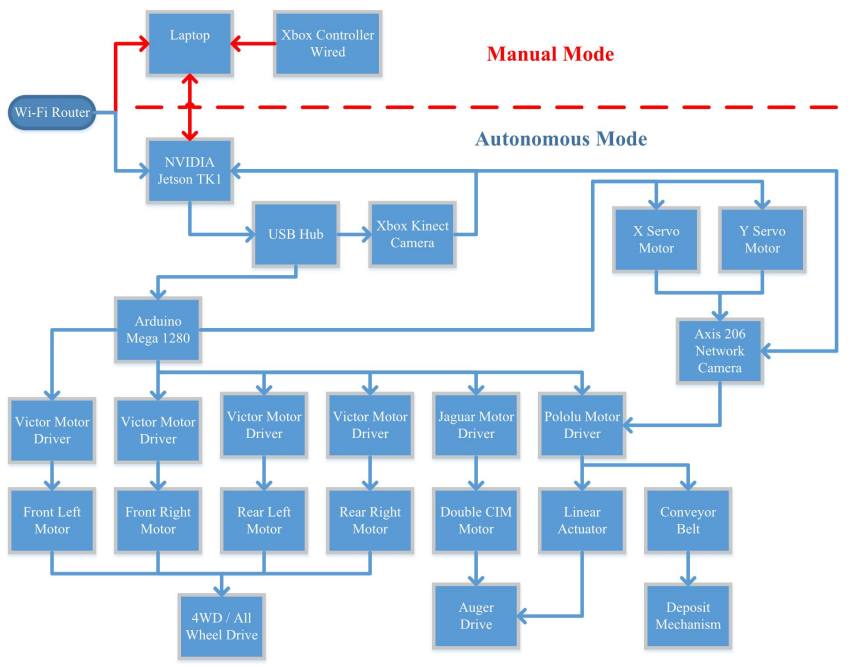

Fig. 11. Circuit System Hierarchy for DustyTRON 2.0

Software team decided to use SSH (Secure shell), which is an encrypted network protocol, and prevent unauthorized access to the Jetson TK1. In addition to using Virtual Network Computing (VNC) communication, bandwidth can also be reduced by compressing the video in the TK1 before being sent to the client computer. The TK1 will use a USB port for both serial communication and powering the Arduino, which will eliminate the need for an extra battery for the Arduino. When the Kinect creates a 3D environment for the TK1, the Kinect will allow the code to choose the shortest path to the target by using the "A Star Algorithm" and drive around any obstacles processes, Fig. 12 is showing the software system hierarchy.

Autonomous C++ code will be built in order to send commands to Arduino and control all motors for a successful move through the Martian simulated terrain; Fig. 13 represent the control setup for the Arduino microprocessor.

There were three types of interfaces that occurred; mechanical interface, electrical voltage/current interface, and data/digital interface. The first one is the hardware components interacting with each other such as gears and chains. The second is hardware components communicating with electrical component which will be dominate with electrical voltage/current signal. The third interface is digital data which occurs between the GPU/processor, sensor, and cameras. Some of the interfaces that will be utilizing for the software devel-

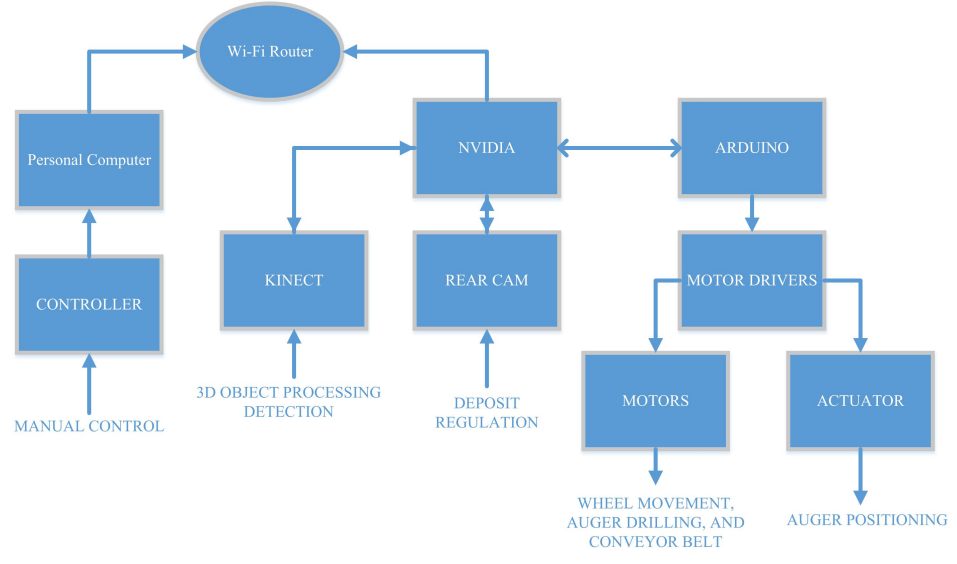

Fig. 12. Software System Hierarchy for DustyTRON 2.0

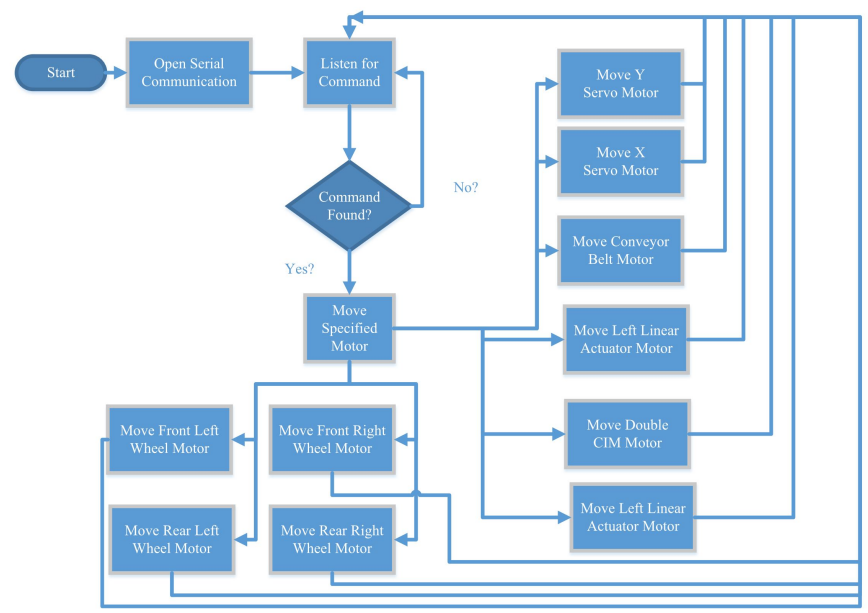

Fig. 13. Arduino Control System Hierarchy for DustyTRON 2.0

opment of the robot include Ubuntu 14.04, Arduino Software IDE and Visual-Basic. The Ubuntu interface was used in order to install, update, and operate the Jetson TK1. The Arduino Software interface was used in order to code the Arduino Mega. Visual-Basic was used in order to program the control configuration for both of the wired Xbox 360 controllers. These interface and interactions between all DustyTRON 2.0 robot's systems is displayed in Fig. 14.

\section{Risk Management, Trade-OfF Assessments And REQUIREMENTS VERIFICATION}

To be prepared in case anything does not go according to plan, the team came up with various ways the robot would fail. Each failure was ranked according to the consequences on the overall performance of the robot, also, each failure is given a likelihood of happening.

\section{A. Hardware Team}

1) Risk Management: Fig. 15 was made to illustrate both the importance and probability of each failure (risk) for robot hardware design during or before the competition: 


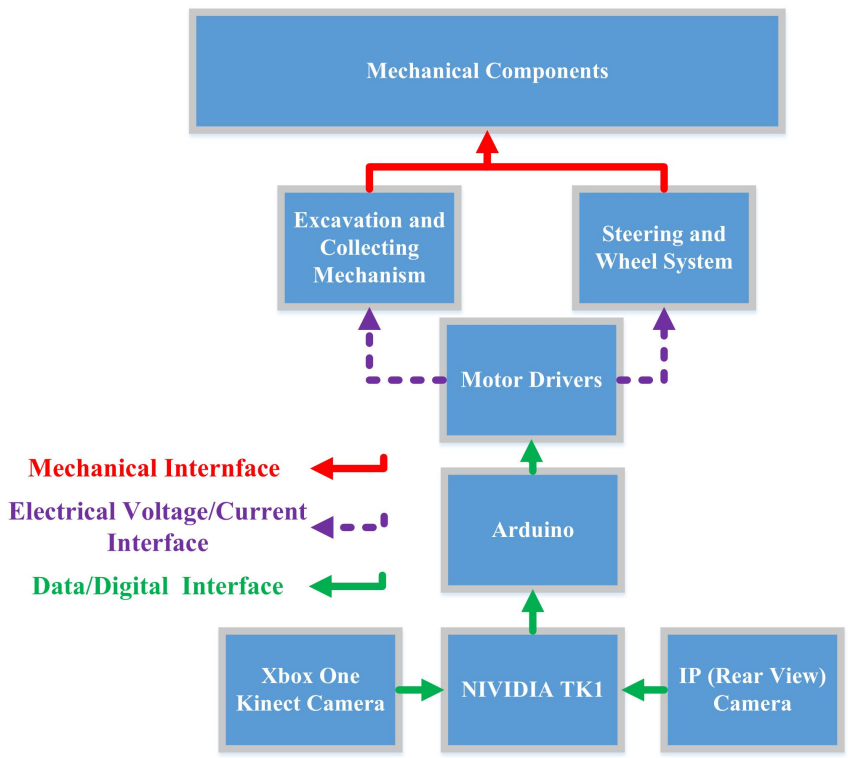

Fig. 14. Interaction Hierarchy between DustyTRON 2.0 Systems

- Failure of the excavation system: If the excavation system is not able to function, or fails to excavate enough regolith to satisfy the requirements.

- Frame integrity failure: If the frame is not able to support the weight of the auger, electrical component box, or the weight of the collected regolith.

- Failure to collect regolith: This might happen if the conveyor belt is not able to hold the regolith securely, or if the auger fails to deliver the regolith to the conveyor belt.

By categorizing the type of risks, and the possibility of them happening, the team will be able to better adapt to the failures and have be prepared to fix any issues that might arise.

\begin{tabular}{|c|l|l|l|l|l|}
\hline \multirow{2}{*}{ Likelihood } & \multicolumn{5}{|c|}{ Consequences } \\
\cline { 2 - 6 } & Insignificant & Minor & Moderate & Major & Catastrophic \\
\hline Almost Certain & & & & & \\
\hline Likely & & & & & \\
\hline Moderate & & & & Failure to collect & \\
\hline Unlikely & & & & Excavation failure & \\
\hline Rare & & & & & Frame Failure \\
\hline
\end{tabular}

Fig. 15. DustyTRON 2.0 Hardware Risk Management

2) Trade-off Assessment: Quality Functional Deployment (QFD) is a focused methodology that takes into account the voice of the customer and develop a response to those needs and expectations. The customer is NASA and they provided the team with their voice as rules and regulations, the team developed few capabilities that can be controlled to achieve the needs. Fig. 16 shows the QFD developed including a list of needs was generated based on the criterion that was set by NASA's Robotic Mining Competition. Then a list of specifications based on these needs and their importance was created and our team goals were included and prioritized according to their importance based on the performance improvement required. This method allowed the team to weight their designs and found which one should be selected which was our final design.

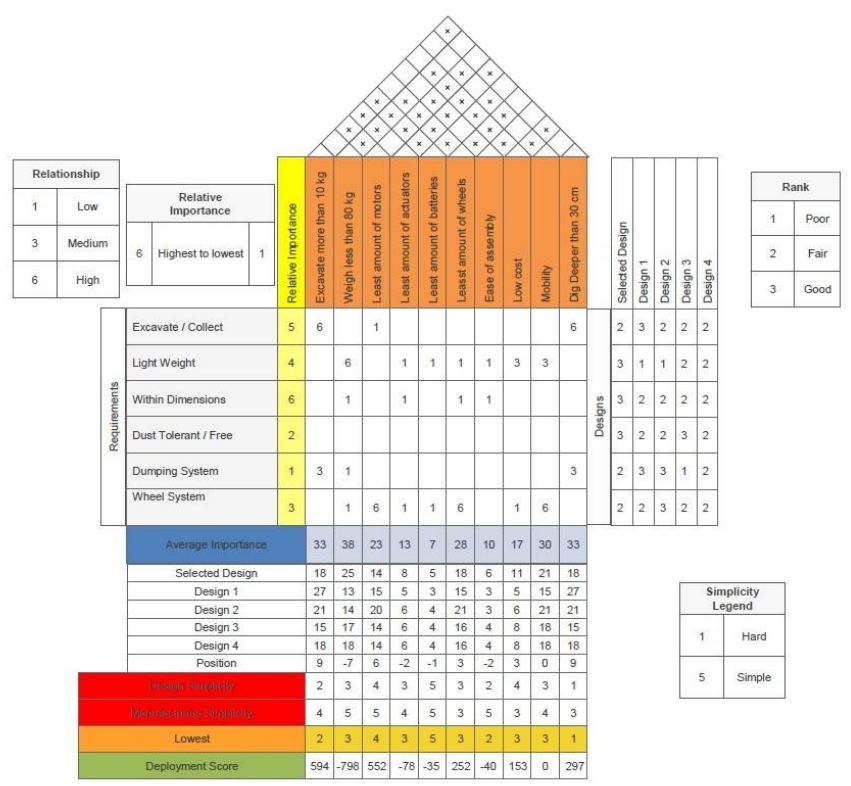

Fig. 16. DustyTRON 2.0 Quality Function Deployment (QFD)

Comparing the critical design to preliminary designs, robot dimensions/weight was ranked the highest priority task. Therefore, an option of balancing the NASA RMC constraints and the team goals were selected. Some of the trade-off for the hardware team include not using an adaptive suspension system to change the robot height and stabilize the robot frame on a rough terrain, because of the added weight. Also, switching from a bigger collection box to conveyor system to simplify the electrical-mechanical components.

\section{B. Electrical Circuit Team}

1) Risk Management: Circuits can be very delicate but can be made to withstand the system requirements. As with every component of the robot, circuit design was also taken into consideration for any possible failures. Fig. 17 categorizes the possible circuit risks for the robot:

- Battery Failure: If one of the batteries fails to provide the required voltage/current.

- Connection Failure: If connections get lose or overheat.

- Motor Driver Failure: If motor driver is not powering its assigned motor any longer.

- Motor Failure: If the motor malfunctions and will not be able to drive the necessary component (wheel or auger).

Because these problems may show during the building process, circuit team prepared for every scenario during the 


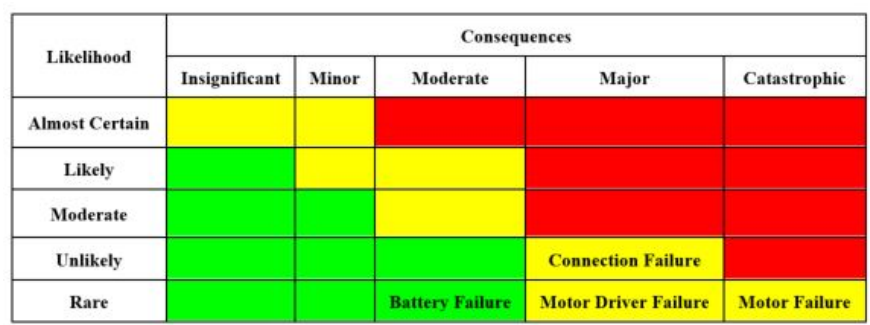

Fig. 17. DustyTRON 2.0 Electrical Circuit Risk Management

testing phase to prevent any future possible problem. Selecting compatible components, and verifying its operation which includes correct wiring under the correct conditions, the probability of risk is greatly reduced. However, in case of unfortunate situation of a component breaking down, team designed the circuit in a simple way that will allow easier components replacement.

2) Trade-off Assessment: The first trade-off was the power source, originally, the robot was running a high discharge $22.2 \mathrm{~V}$ (5000 mAh) 6-cell battery which was super light (1.5 lb.) but it was extremely powerful and was able to burn several motor drivers. Therefore, team decided to go with multiple lower power batteries even if they are heavier (16 lb.) and required more connections. The second major trade-off was the motor drivers, the first selected type was an easy to plug and play devices but they have a limited performance. The team opt to use different brand which required soldering and configuration the motor driver to the required setting because of the superior performance.

\section{Software Team}

1) Risk Management: Software failure can be due to either connection of related-hardware failure. Software team failures might be:

- $\quad$ Failure of NVIDIA TK1 power regulator: If power regulator of the TK1 is not able to function properly, the TK1 will be damaged and not work at all.

- $\quad$ Failure of VNC connection: If the VNC connection were not working properly, no rapid maintenance will be provided to the TK1 thus a risk for potential inoperability.

- $\quad$ Failure of programming Open-CV: If Open-CV library were not configured or programmed correctly, the robot autonomous mode will be disabled.

- Failure to send command to Arduino: If we were to run out of characters to program each movement of the robot, we would not be able to move the robot's individual components.

- $\quad$ Failure to have available PWM pins in Arduino: If we run out of PWM pins, we would have to change the code to fit analog and digital pins instead.

These failures had been illustrated in Fig. 18.

\begin{tabular}{|c|c|c|c|c|c|}
\hline \multirow{2}{*}{ Likelihood } & \multicolumn{5}{|c|}{ Consequences } \\
\cline { 2 - 6 } & Insignificant & Minor & Moderate & Major & Catastrophic \\
\hline Almost Certain & & & & & \\
\hline Likely & & & & & \\
\hline Moderate & & & & & \\
\hline Unlikely & & & & Connection Failure & \\
\hline Rare & & & Battery Failure & Motor Driver Failure & Motor Failure \\
\hline
\end{tabular}

Fig. 18. DustyTRON 2.0 Software Risk Management

2) Trade-off Assessment: Software team had a major tradeoff issue by using Jetson TK1 with lower processing power over Jetson TX1 because TX1 was back-ordered. For manual control, they opt to use Xbox 360 wired controllers to avoid any lag issues and wire management will be required. In addition, two Xbox 360 controllers will be used, where one person can excavate while the other person operates the robot. This will limit the controller buttons mapping errors and reduce the human error. The button layout of the Xbox 360 controller is arguably the best ergonomic and user friendly in the market. Arduino Mega 1280 was used over Arduino Mega 2560, because Arduino Mega 1280 is available from last year which will reduce the budget. This trade-off resulted in reducing the available memory but the robot gained an extra $20 \mathrm{~mA}$ DC current per I/O pin. Arduino Mega will be used for Pulse-Width-Modulation (PWM) instead of Jetson TK1 because Arduino is more stable. Xbox Kinect Camera was selected over other cameras because of its 3D processing, sensors, and image detection capabilities, which will benefit in achieving the autonomous mode. Axis 206 Camera was used over other cameras because it comes with two servo motors to control $\mathrm{x}$ - and $\mathrm{y}$-axis.

\section{Verification of System Meeting Requirements}

To create DustyTRON 2.0, the team felt the robot had to meet certain specific requirements which were both teamoriented and NASA RMC derived. The following requirements were taken into consideration.

1) Functional Requirements: Robot will be able to excavate the simulated Martian terrain.

- Robot shall be able to operate by tele-robotic operations or autonomously to reach its destination.

- Collected material shall be deposited and stored into the conveyor belt until the deposition.

- The robot shall travel the arena to the collector bin and if needed return to the mining area to continue excavating.

- When collecting and depositing the obtained BP-1, robot shall be able to evade the obstacle presented in the arena.

- Easier and understandable code shall be established for the usage of controllers. 


\section{2) Performance Requirements:}

- Robot shall be able to collect $10 \mathrm{~kg}$ of BP-1 to deposit in the collector bin and shall be able to operate for 10 continuous minutes.

- The BP-1 excavated and collected shall be obtained from opposite end of the arena from where the robot is placed and robot shall begin excavating once it reaches a mining line.

- Robot shall incorporate a protective mechanism to shield the electronics parts and avoid picking up excess amount of dust.

- The bandwidth that the robot consumes during communication functions shall be reduced.

\section{3) Physical Requirements:}

- Mining robot shall weigh a maximum of $80 \mathrm{~kg}$ including any subsystems and cameras.

- $\quad$ Robot shall be self-powered with on-board power and the energy used shall be recorded.

- Initial dimensions of the robot shall be within $1.5 \mathrm{~m}$, $0.75 \mathrm{~m}$, and $0.75 \mathrm{~m}$ in length, width, and height, respectively.

4) Safety Requirements:

- An emergency red button will be installed on the robot to terminate its actions. The button shall be $40 \mathrm{~mm}$ in diameter or greater and should be easily accessible.

- The electrical wiring must be correctly installed with the appropriate connections to avoid any accidents.

- An easy connection setup between the robot and the control station, while keeping a high level of security, shall be incorporated.

To ensure that the robot's system is meeting all necessary requirements, the team must follow NASA RMC's regulations and guidelines. Verification started by testing and inspecting each component for defects. Commercial off-the-shelf (COTS) parts were tested before used on the robot to verify their performance and specifications. Some of the actions that DustyTRON 2.0 team followed are:

- The extruded T-slot bars' integrity was inspected for any damage or defects before constructing the frame. Fitting and testing bar ability to support the weight of the excavation mechanism and its vibration.

- Twin spiral auger was tested in the simulated field, to make sure it would reach an adequate depth in order to reach the ice region while being able to excavate an adequate amount of regolith.

- Linear actuators were tested to be able to lift and push the weight of the auger mechanism and frame.

- Excavating motor was tested to make sure it can produce enough torque to satisfy the excavating needs, break through the surface of the simulated terrain, and handle the ice-simulated material.
- Conveyor belt was verified to be strong and sturdy to not come apart once it starts moving while carrying the regolith or ice simulant. Also, the smooth and stable movement of the conveyor rollers and motor was tested.

- Wheels and their motors were tested on sand in order to verify the ability to maneuver effectively through such terrain carrying the robot and collected regolith weight.

- Batteries were tested to make sure that they have the required power to operate the robot with all of its components at maximum capacities for more than 10 minutes.

- Emergency-stop buttons were tested to verify if they could cut off the batteries' power from the whole robot when needed.

- Power consumption analyzer were tested to confirm its calibration status.

- 4-Wheel system was programmed and tested using the selected motor drivers and the Xbox controllers.

- Axis 206 Network Camera were tested and verified that it can reach the 180 degrees rotation limit while broadcasting the live feed to NVIDIA TK1.

- Xbox Kinect V2 camera functionally was validated by processing live images and to detect object depth perception.

- Stable VNC connection over the WiFi was established and tested to allow a complete control and monitoring of the TK1 through a laptop to provide maintenance when needed.

\section{E. Reliability}

To ensure the maximum reliability of the robot, DustyTRON 2.0 team has taken precautions in solving the potential issues from last year's robot, which will help to elongate the robot's Mean Time Between Failures (MTBF). Hardware team focused on building a frame that is reliable enough to support the excavation mechanism, along with the collected regolith weight, while still being able to move around efficiently by selecting better wheels to maneuver in the uneven simulated terrain during the competition.

For the circuit team, reliability will be increase by reducing the amount of cables and connection used, because less components means less probability of having a breakdown. The second task is securing and protecting the cabling from harm or damaging elements. Cable management will be taken into account by using power distributor board with attached voltage regulator, which will eliminate the need of any unnecessary connection with multiple voltages. The robot's power source will be four batteries which are connected in serial/parallel configuration what will assure a stable voltage/current while discharging all batteries evenly at the same time. One more measure to make the system more reliable was placing the circuitry box in an easily accessible area which will help in the case of diagnostic and repair. 
To address the reliability issues within the robot software, the first action is to maintain a strong connection between the Jetson TK1 and the Arduino. Using USB serial communication will pass commands and power Arduino at the same time. Also, establishing a VNC connection can help in immediately addressing any problems within the TK1 and fix it by using a windows-based laptop. The VNC connection will be a SSH connection so that no one can interfere with the robot communication. Another issue was solved by using Arduino Mega, instead of the Jetson TK1, to establish the Pulse Width Modulation (PWM) of the robot as the PWM in Arduino Mega is more accurate and more reliable which will result in making commands and controls smoother with no lag, Appendix $\mathrm{C}$ is showing the updated code for the Arduino.

\section{COMPETITION RESUlTS SUMmaRY}

After several designs and modifications, the team was able to develop a new robot design that met the NASA RMC regulations and delivered the DustyTRON 2.0 robot to Kennedy space Center in Florida, shown in Fig. 19 and 20. The robot passed all inspections steps and competed against 56 other robots from all over United State and placed 16th. This experience was exceptional as the team members were able to implement all their engineering knowledge and skills to contribute to the Space exploration mission.

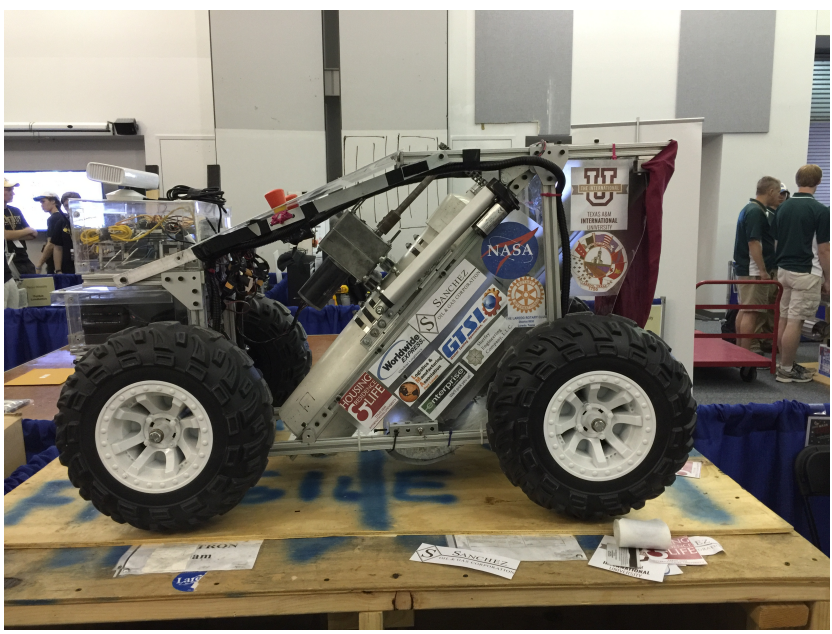

Fig. 19. DustyTRON 2 Robot - Final RMC 2016

\section{CONCLUSION}

In conclusion, DustyTRON team shown in Fig. 21 was created in August 2014, the team members were set to showcase their skills and utilize their knowledge in a stateof-art challenge. The NASA RMC was a great opportunity for them and placing the 16th out of 56 invited universities, was a respectable performance for a second-year team. The completed a new robot design that signifies the combination of mechanical, electrical, and computer engineering and computer science disciplines integrated into one cohesive system and present the power of their Systems Engineering background. The team worked on a completely new design being resourceful and utilize all the available sponsors and learned invaluable lessons about systems engineering principles and their implantation in a real-file problem solution.

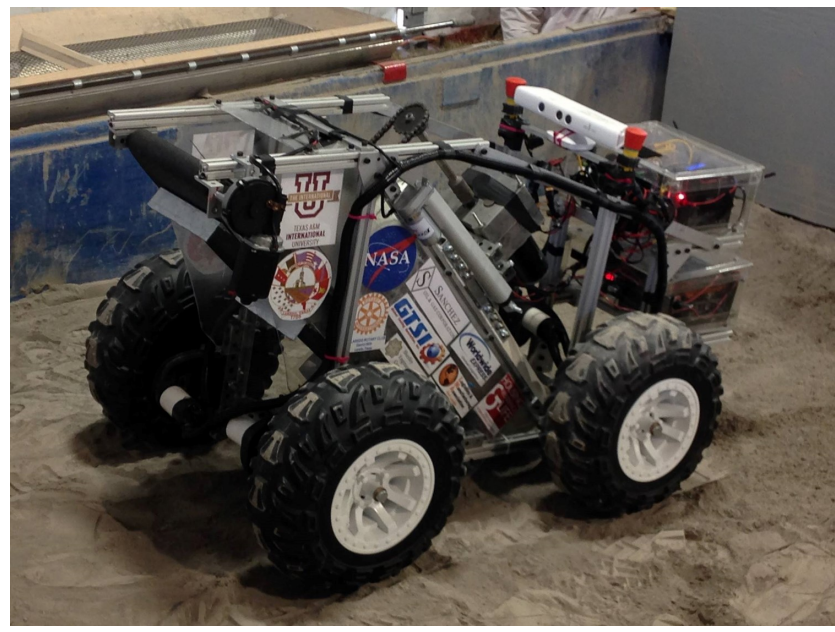

Fig. 20. DustyTRON 2 Robot - In Action

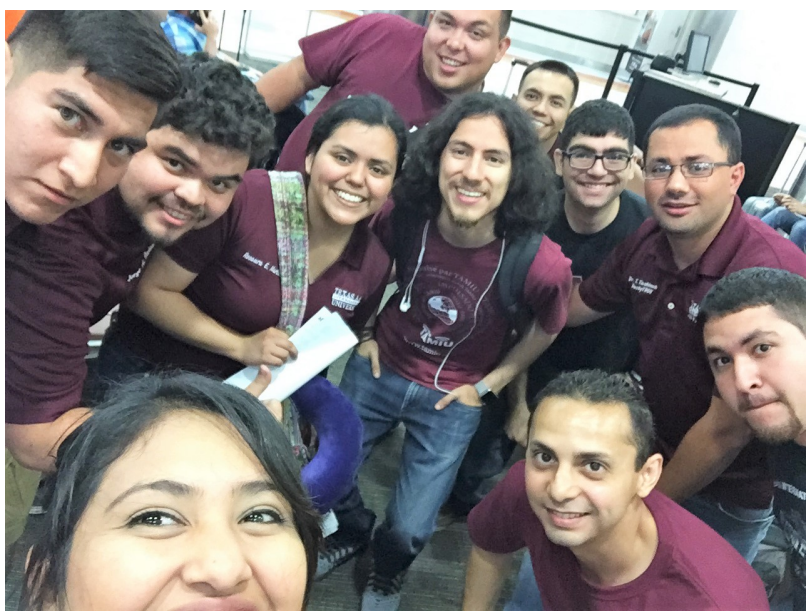

Fig. 21. DustyTRON 2 Team

The robot's locomotion system proved to be robust to handle the rough terrain that was encountered without failures, while staying under the expected weight and within a reasonable budget. One of the lesson learned from the project was the importance of time and team management, working to achieve all the goals taking into account the time and regulation constraint.

The team hopes to use and improve the robot for future competitions, therefore some of the improvements that can be tackled:

- Further develop the autonomy operation and enhance the robot vision software. The robot currently utilized partial autonomy to control its digging and dumping actions.

- Improve the sliding auger system, as it showed a significant success in digging and collecting regolith.

- Enhance the adaptive suspension system and improve the steering system, the robot has eclectically controlled suspension and drifting style steering system.

The team will continue to be involved with the local 
community outreach by presenting to local school to promote interest in the field of robotics, mainly NASA's current programs and projects in robotics. The team had presented to local middle and high school students their robotics projects to encourage them to join the STEM fields. Additionally, the team had been actively mentoring local FIRST Lego/Tech/Robotics teams in both stages of building and programming of their own robot, and host these competitions locally.

\section{ACKNOWLEDGMENT}

Thanks to NASA for providing such a great opportunity to participate in such competition. This project would not be successful without the support of Texas A\&M International University (TAMIU) and our sponsors from Laredo, TX.

\section{REFERENCES}

[1] Voosen, Paul. "Mars rover steps up hunt for molecular signs of life." (2017). Science 03 Feb 2017, Vol. 355, Issue 6324, pp. 444-445, DOI: $10.1126 /$ science.355.6324.444

[2] Koris, Daniel R., and Jason Isaacs. (2017) "A Formal Approach to Extended State Machines for Multi-Objective Robots Operating in Dynamic Environments." Proceedings of the 2017 Midstates Conference on Undergraduate Research in Computer Science and Mathematics

[3] Tashtoush, T., Hernandez, R., Yanez, R., Gonzalez, J., Moreno, H., and Escobar, V. (2020). "Reverse-Twister Swarm Search Algorithm Design: NASA Swarmathon Competition", International Journal of Research Studies in Computer Science and Engineering (IJRSCSE), 7(1), pp.1320.

[4] Hernandez, R., Yanez, R., Gonzalez, J., Moreno, H., Escobar, V., and Tashtoush. T., (2016) "Design of a Swarm Search Algorithm: DustySWARM Reverse-Twister Code for NASA Swarmathon.” Texas A\&M International University, School of Engineering.

[5] Tashtoush, T., Gutierrez, O., Herrera, E., Medina, J., Peña, A., Varela, E., and Hernandez, R. (2020). "Design of a Swarm Search Algorithm: DustySWARM Spiral Epicycloidal Wave (SEW) Code for NASA Swarmathon", International Journal of Research Studies in Computer Science and Engineering (IJRSCSE), 7(1), pp.28-36.

[6] Gutierrez, O., Herrera, E., Medina, J., Peña, A., Varela, E., Hernandez, R., and Tashtoush. T. (2017) "Design of a Swarm Search Algorithm DustySWARM Spiral Epicycloidal Wave (SEW) Code for NASA Swarmathon". Texas A\&M International University, School of Engineering.

[7] Tashtoush, T., Ruiz, C., Estevis, T., Herrera, E., Bernal, R., Martinez, R., and Reyna, L. (2020). "Square Spiral Search (SSS) Algorithm for Cooperative Robots: Mars Exploration", International Journal of Research Studies in Computer Science and Engineering (IJRSCSE), 7(1), pp.21-27.

[8] Tashtoush, T. H., Hernandez, R., Yanez, R., Gonzalez Jr, J., Moreno, H., \& Escobar, V. (2020). Reverse-Twister Swarm Search Algorithm Design: NASA Swarmathon Competition. International Journal of Research Studies in Computer Science and Engineering (IJRSCSE), 7(1), 13-20.

[9] Ruiz, C., Estevis, T., Herrera, E., Bernal, R., Martinez, R., Reyna, L., and Tashtoush, T., (2018) "Design of a Swarm Search Algorithm: Square Spiral Search (SSS) Algorithm for NASA Swarmathon". Texas A\&M International University, School of Engineering.

[10] Secor, P. (2016). "NASA Swarmathon".

[11] Braccio, M. (2019). Design of a Robot for the 2019 NASA Robotic Mining Competition. In Proceedings of the Wisconsin Space Conference (Vol. 1, No. 1).

[12] Neubert, J. J. (2016). Using NASA's Robotic Mining Competition to Give Students a Quality Sys-tems Engineering Experience. In ASEE's 123rd Annual Conference \& Exposition (pp. 4-11).

[13] Guerra,L., Murphy, G., and May. L., (2013). Applying Engineering to the Lunabotics Mining Competition Capstone Design Challenge. Proceeding of the ASEE Annual Conference and Exposition, June 2013.

[14] Stecklein, J. (2017, July). NASA's Robotic Mining Competition Provides Undergraduates Full Life Cycle Systems Engineering Experience. In INCOSE International Symposium (Vol. 27, No. 1, pp. 1456-1473).
[15] Chaput, A., 2016, 'System Engineering Education for All Engineers - A Capstone Design Approach'. ASEE 123rd Annual Conference \& Exposition, New Orleans, June 26-29, 2016.

[16] Mahmood, M. 2016, 'Oakton Community College 2016 NASA Robotic Mining Competition Systems Engineering Paper', paper presented to the 2016 NASA Robotic Mining Competition, Kennedy Space Center, Florida, 16-20 May.

[17] The University of Alabama in collaboration with Shelton State Community College, 2016, 'Journey to Mars; 2016 Systems Engineering Paper', paper presented to the 2016 NASA Robotic Mining Competition, Kennedy Space Center, Florida, 16-20 May.

[18] Charlotte 49er Miner Robotics, The University of North Carolina at Charlotte, 2016, '2016 Systems Engineering Paper', paper presented to the 2016 NASA Robotic Mining Competition, Kennedy Space Center, Florida, 16-20 May.

[19] Illinois Robotics in Space (IRIS), University of Illinois at UrbanaChampaign, 2016, 'Design and Development of the IRIS-6 Robotic Mining System', paper presented to the 2016 NASA Robotic Mining Competition, Kennedy Space Center, Florida, 16-20 May.

[20] John Brown University Eaglenaut Robotics, John Brown University, 2015, 'Robotic Regolith Excavation System', paper presented to the 2015 NASA Robotic Mining Competition, Kennedy Space Center, Florida, 1822 May.

[21] Chicago EDT Robotics, University of Illinois at Chicago, 'Systems Engineering Report 2016, University of Illinois at Chicago, AMES-3 Surus', paper presented to the 2016 NASA Robotic Mining Competition, Kennedy Space Center, Florida, 16-20 May.

[22] Iowa State University Cyclone Space Mining, '2015-2016 Systems Engineering Paper', paper presented to the 2016 NASA Robotic Mining Competition, Kennedy Space Center, Florida, 16-20 May.

[23] Dieter, G. E., \& Schmidt, L. C. (2013). Engineering design. Boston: McGraw-Hill Higher Education.

[24] "Rules and Rubrics", Nasa.gov, 2016. [Online]. Available: http://www. nasa.gov/offices/education/centers/kennedy/technology/nasarmc/RulesR ubricsResources

[25] Kapurch, S. J. (Ed.). (2010). NASA systems engineering handbook. Diane Publishing.

[26] Bellestri, S., Boil, T., Carswell III, M., et al. (2013). Alabama Lunabotic 2013 Systems Engineering Paper (Undergraduate Thesis) Retrieved from NASA.

[27] Alfaro, D., Aranguren, A., Duarte, T., De La Cruz H., Perez, G., Torres, A. Delgado, J., Melero, D., Vazquez, J. A., Charlton, B., Jose Guajardo, J., Flores, G., Garza, E., \& Tashtoush, T. (2015). Systems Engineering Paper (Undergraduate Thesis) Retrieved from Texas A\&M International University School of Engineering.

[28] Ay, N., Bertschinger, N., Der, R., Güttler, F., \& Olbrich, E. (2008). Predictive information and explorative behavior of autonomous robots. The European Physical Journal B, 63(3), 329-339.

[29] Shotts Jr, W. E. (2012) "The Linux command line: a complete introduction" No Starch Press.

[30] Carson, E. M., Rivadeneira, J., Woodward, N. K., \& Peterson, P. W. (2016). "NASA Robotic Mining Competition 2015-2016".

[31] Mueller, R. P. (2012) "Lunabotics Mining Competition: Inspiration through Accomplishment" Thirteenth ASCE Aerospace Division Conference on Engineering, Science, Construction, and Operations in Challenging Environments, and the 5th NASA/ASCE Workshop On Granular Materials in Space Exploration.

[32] Williams, W. B., \& Schaus, E. J. (2015). Design and Implementation of a Rocker-Bogie Suspension for a Mining Robot. In ASEE Southeast Section Conference.

[33] Liu, Y., Jeremy B., Zachary C., Jennifer B., John A.s, Madelyn D., David S., Cindy L. B., John B., and Christopher A.. "Mechanical design, prototyping, and validation of a Martian robot mining system." SAE International Journal of Passenger Cars-Mechanical Systems 10, no. 2017-01-1305 (2017): 177-182.

[34] Mueller, R., \& Van Susante, P. (2011, September). A review of lunar regolith excavation robotic device prototypes. In AIAA SPACE 2011 Conference \& Exposition (p. 7234). 